\title{
Alergia a anestésicos locales y generales y a otros medicamentos durante procedimientos quirúrgicos
}

\author{
María Claudia Ortega ${ }^{1}$, Santiago López ${ }^{2}$, Fabio F. Morato ${ }^{3}$, Luciana Kase Tanno ${ }^{4}$, \\ Luis Felipe C. Ensina ${ }^{5}$
}

\section{Resumen}

Las reacciones de choque e hipersensibilidad a los anestésicos locales y generales y a otros medicamentos utilizados durante los procedimientos quirúrgicos, continúan siendo un reto en la práctica clínica. Las reacciones de hipersensibilidad alérgica pueden variar en su presentación e intensidad, y pueden producir desde síntomas leves en la piel hasta la muerte.

Palabras clave: hipersensibilidad, anestésicos, alergia.

\section{Title}

Allergy to local and general anesthetics and to other drugs during surgical procedures

\begin{abstract}
Shock and hypersensitivity reactions to local and general anesthetics and to other drugs used during surgical procedures continue being a challenge in clinical practice. Allergic hypersensitivity can vary in presentation and intensity and might manifest itself from mild cutaneous symptoms to death.
\end{abstract}

Key words: hypersensitivity, anesthesia, allergy.

1 Médica pediatra, alergóloga, inmunóloga clínica; Gestión Aplicada a los Servicios de Salud, Hospital Universitario San Ignacio; profesora asistente, Departamento de Pediatría, Pontificia Universidad Javeriana, Bogotá, D.C., Colombia.

2 Médico especialista en Alergología e Inmunopatología Clínica, Hospital de Clínicas, Universidad de São Paulo, Brasil; Hospital Infantil Los Ángeles, San Juan de Pasto, Colombia.

3 Profesor asociado y libre docente, Inmunología Clínica y Alergia, Facultad de Medicina, Universidad de São Paulo; supervisor, Servicio de Inmunología Clínica y Alergia, Hospital de Clínicas, São Paulo, Brasil.

4 Médica especialista; colaboradora, Servicio de Inmunología Clínica y Alergia, Hospital de Clínicas, São Paulo, Brasil.

5 Médico especialista; colaborador, Servicio de Inmunología Clínica y Alergia, Hospital de Clínicas, São Paulo, Brasil.

Recibido: 01-02-2010

Revisado: 22-05-2010

Aceptado: 24-06-2010

Ortega M. C., López S., Morato F., Kase Tanno L., C. Ensina L. F., Alergia a anestésicos locales y generales y a otros... 


\section{Introducción}

Las reacciones adversas a los fármacos utilizados durante los procedimientos quirúrgicos han sido estudiadas y notificadas desde hace treinta años. Todavía son causa de gran preocupación por parte de los profesionales de la salud.

Las reacciones adversas a los fármacos son definidas como cualquier efecto "no terapéutico" en las dosis habitualmente empleadas para la prevención, diagnóstico y tratamiento de las enfermedades[1]. Pueden dividirse en los siguientes dos grandes grupos.

Reacciones prevenibles. Se asocian con los efectos conocidos del medicamento; dependen de la dosis, se presentan en individuos, generalmente, normales y corresponden al $80 \%$, más o menos, de los efectos secundarios de los medicamentos. Incluyen toxicidad, efectos colaterales e interacciones de los medicamentos.

Reacciones no prevenibles. En general, no dependen de la dosis y no están relacionadas con el efecto farmacológico del medicamento. Están implicados factores propios del paciente, como alteraciones inmunológicas y de predisposición genética. $\mathrm{Se}$ manifiestan como reacciones idiosincrásicas, intolerancia, reaccio- nes de hipersensibilidad alérgicas y no alérgicas.

Las reacciones de hipersensibilidad se definen como cualquier reacción iniciada por un estímulo determinado, que se puede reproducir. Se considera alérgica cuando existe de por medio un mecanismo inmunológico $\mathrm{y}$, no alérgica, cuando no hay un mecanismo inmunológico asociado.

Hipersensibilidad alérgica. Ocurre en un pequeño número de pacientes $\mathrm{y}$ es responsable de cerca de $6 \%$ de todas las reacciones adversas[2]. Es imprevisible, no se correlaciona con los efectos farmacológicos del medicamento y no depende de la dosis.

Es necesario que haya una exposición previa al medicamento o a una sustancia inmunológicamente relacionada con la formación de anticuerpos específicos.

Las presentaciones clínicas pueden clasificarse según el mecanismo fisiopatológico relacionado.

Hipersensibilidad no alérgica. Se caracteriza por una reacción inmediata con degranulación de mastocitos y liberación de mediadores por un mecanismo independiente de la $\operatorname{IgE[2]}$. Ocurre sin exposición previa y mimetiza una reacción de tipo I, pero no hay presencia de IgE específica. Se 
produce por la activación directa del medicamento en la superficie de los mastocitos y no difiere clínicamente de las reacciones de hipersensibilidad alérgica. La producen, por ejemplo, los opiáceos, la vancomicina y la polimixina $\mathrm{B}$.

\section{Epidemiología}

La prevalencia exacta de la anafilaxia durante los procedimientos quirúrgicos no está bien determinada. Esto se debe a métodos deficientes de recolección de datos, a la dificultad en el reconocimiento de las reacciones por parte de los profesionales de la salud, y a la falta de pruebas cutáneas y de laboratorio estandarizadas que diferencien entre reacciones anafilácticas y anafilactoides y que sirvan, a su vez, para identificar el factor etiológico.

La incidencia estimada de anafilaxia intraoperatoria en Francia varía de 1 en 3.500 a 1 en 13.000 procedimientos y, en Australia, de 1 en 10.000 a 1 en 20.000 procedimientos[3, 4].

Las manifestaciones varían en intensidad y, frecuentemente, son reacciones inmediatas generalizadas, caracterizadas por broncoespasmo e hipotensión. Las manifestaciones cutáneas o quejas, como el prurito, son poco observadas y poco notificadas en cirugías generales, en las cuales el paciente recibe sedación[1].
La mortalidad es de cerca de $3 \%$ a $6 \%$, con riesgo adicional de $2 \%$ en pacientes con daño neurológico previo.

De los medicamentos, los relajantes musculares son los principalmente implicados en las reacciones anafilácticas intraoperatorias, responsables de $50 \%$ a $70 \%$ de los casos[3,5,6]. El látex es la segunda causa más común de estas reacciones en la mayoría de los estudios, seguido por los antibióticos y los agentes inductores. Los coloides, los opioides y los medios de contrastes son responsables de menos del $10 \%$ de las reacciones.

Es importante recordar que la prevalencia de los factores etiológicos puede variar de acuerdo con la población estudiada.

En relación con los anestésicos locales, las reacciones de hipersensibilidad alérgica son raras. La mayoría de las reacciones descritas con el uso de este grupo farmacológico se manifiesta por reacciones cutáneas de contacto, reacciones de toxicidad, efectos simpaticomiméticos y reflejos vasovagales, por su asociación con vasoconstrictores $[5,7,8]$.

\section{Factores de riesgo}

El diagnóstico de las reacciones adversas a los anestésicos generales y locales se basa principalmente en el detalle de la anamnesis. En ella se de- 
ben caracterizar los factores de riesgo en forma individual[9].

a) $\operatorname{Sexo}$

- El sexo femenino parece ser el más afectado[10].

b) Edad

- Los niños presentan menor sensibilización a los medicamentos que los adultos.

- La edad avanzada presenta un mayor riesgo de reacciones, por las posibles enfermedades concomitantes.

c) Condiciones clínicas del paciente y enfermedades concomitantes[11]

- Atopia respiratoria

- Aumenta la gravedad de las reacciones, principalmente si el $\mathrm{VEF}_{1}$ es menor de 70\%[10].

- Enfermedad cardiovascular concomitante[11].

- Aumenta el riesgo de eventos graves durante los procedimientos.

- El uso de medicamentos como â-bloqueadores e inhibidores de la enzima convertidora de angiotensina (IECA), puede perjudicar la respuesta terapéutica a la reanimación durante una reacción anafiláctica.

- Mastocitosis.

- Es un factor de riesgo aislado para cualquier procedimiento.
Los pacientes deben tener orientación y preparación previas a la anestesia, y se deben usar medicamentos profilácticos[12].

- VIH.

- Aumenta en $100 \%$ la posibilidad de reacciones secundarias a los medicamentos.

- Múltiples procedimientos quirúrgicos aumentan las reacciones de alergia al látex.

d) Factores genéticos

- La velocidad del metabolismo del medicamento influye en la prevalencia de sensibilización (acetiladores lentos).

- Existe relación del HLA en casos de alergia al látex.

- Las malformaciones urogenitales y la espina bífida requieren frecuentemente procedimientos quirúrgicos y utilización de sondas, lo que aumenta la sensibilización al látex[10-13].

- La incidencia familiar está bien demostrada en casos de hipersensibilidad a los antiinflamatorios no esteroideos utilizados durante las intervenciones quirúrgicas o durante el posoperatorio.

e) Reacción previa a medicamentos

- Es el factor de riesgo más importante. Debe tenerse en cuenta que la exposición a grupos 
químicos semejantes puede producir reacciones cruzadas[10].

f) Naturaleza del medicamento

- Las macromoléculas y los medicamentos heterólogos, antígenos complejos, presentan mayor potencial de sensibilización[9].

g) Forma de exposición

- La aplicación tópica cutánea es la forma que presenta mayor riesgo de sensibilización, por ejemplo, sulfas, penicilinas y antihistamínicos.

- La forma de aplicación intravenosa presenta el cuadro de mayor gravedad en las reacciones.

- La velocidad de infusión influye, por ejemplo, en el síndrome del hombre rojo inducido por la infusión rápida de vancomicina[5].

- Los coadyuvantes pueden también ser sustancias sensibilizadoras.

\section{Agentes responsables de anafilaxia durante la anestesia y los procedimientos médicos}

\section{Relajantes musculares}

Los agentes bloqueadores neuromusculares, o simplemente relajantes musculares, son fármacos utilizados frecuentemente en procedimientos de anestesia general y les corresponden de $50 \%$ a $70 \%$ de los casos de anafilaxia durante una cirugía[1].

Todos los relajantes musculares pueden causar anafilaxia y pueden inducir dos tipos de reacción: una mediada por IgE, relacionada con la estructura de amonio, y otra no inmunológica (seudoalérgica o anaflactoide), por estimulación no específica de los mastocitos.

Laxenaire et al. estudiaron 467 pacientes entre enero de 1997 y diciembre de 1998 y observaron que la succinilcolina y el rocuronio eran los medicamentos principalmente involucrados, seguidos por el vecuronio y el pancuronio. El atracuronio fue el relajante muscular menos relacionado con las reacciones secundarias.

La prevalencia de reacción cruzada entre los relajantes musculares es de $65 \%$ por la prueba cutánea y de $80 \%$ por radioinmunoensayo.

La reacción cruzada depende de la configuración del parátopo del anticuerpo, que puede corresponder apenas al epítopo o se extiende por regiones adyacentes de la molécula. No se refiere a la flexibilidad estructural ni a la distancia entre las moléculas de amonio ni a la afinidad relativa de los diferentes relajantes musculares a la IgE específica[3]. 
El diagnóstico de las reacciones por relajantes musculares se basa en la historia clínica completa y detallada, y se apoya en los resultados de las pruebas cutáneas. La prueba cutánea de puntura se hace, generalmente, con el medicamento puro, sin diluir, con excepción de la succinilcolina, el atracurio y el mivacurio. Las concentraciones no irritantes para la práctica de las pruebas cutáneas de lectura inmediata han sido determinadas pero existen variaciones de protocolos en la literatura.

Una vez que la prueba de puntura se reporta como negativa, debe considerarse practicar la prueba intradérmica en diluciones inicialmente de $1 /$ 1.000 a 1/100. La concentración del medicamento debe --aumentarse progresivamente si los resultados son negativos.

A pesar de ser muy confiables, las pruebas cutáneas no demuestran eficiencia diagnóstica absoluta[3].

Entre las pruebas in vitro, la dosificación de IgE específica no está disponible, excepto para el suxametonio (succinilcolina) (Phadia c202). Sin embargo, la sensibilidad de la prueba aún es baja (30\% a 60\%).

La prueba de activación de basófilos, a pesar de no estar estandarizada, mostró una sensibilidad mayor de $60 \%$ y especificidad de $90 \%$, y con- tribuye de manera importante en el diagnóstico de las reacciones por relajantes musculares[3].

La investigación de la hipersensibilidad a los relajantes musculares sólo se justifica en aquellos individuos que hayan presentado alguna reacción grave en procedimientos anestésicos previos. En estos casos, la prueba cutánea debe practicarse, no sólo para confirmar el diagnóstico, sino, también, para determinar una alternativa segura[5]. No existen pruebas que demuestren un menor riesgo de reacción en los individuos que reciben medicación previa con corticosteroides, antihistamínicos o ambos[1-14].

\section{Hipnóticos}

La incidencia estimada de reacciones anafilactoides con el tiopental es de 1:30.000[5] Existen pruebas de que la mayoría de las reacciones son de origen seudoalérgico. Sin embargo, ya se han descrito casos de reacciones alérgicas verdaderas mediadas por IgE y se cuenta con algunos protocolos para practicar pruebas cutáneas de lectura inmediata con concentraciones no irritantes del medicamento[5].

\section{Opioides}

Los analgésicos opioides inducen liberación de mediadores por los mastocitos, de forma directa, no me- 
diada por factores inmunológicos. Los estudios muestran que solamente los mastocitos cutáneos son sensibles a los opioides; por lo tanto, la reacción parece ser autolimitada y restringida especialmente a la piel, con síntomas como prurito y urticaria, y algunas veces, hipotensión leve[1-9].

Las pruebas cutáneas con codeína, morfina o meperidina, reportan una reacción positiva en prácticamente todos los seres humanos, produciendo la activación directa de los mastocitos[5]. No se encuentran disponibles comercialmente pruebas diagnósticas in vitro.

\section{Antiinflamatorios no esteroideos (AINE)}

No hay un mecanismo inmunológico comprobado para la mayor parte de las reacciones adversas a los antiinflamatorios no esteroideos y analgésicos. Se cree que esta clase farmacológica inhibe la enzima cicloxigenasa, responsable de una de las vías del metabolismo del ácido araquidónico. De esta forma, existe un aumento en la síntesis de leucotrienos, sustancia responsable de los síntomas más frecuentes de estas reacciones de hipersensibilidad no alérgica: urticaria, angiedema, broncospasmo y, en casos graves, choque[5].

La mayoría de los AINE posee el mismo mecanismo de acción, con la aparición frecuente de reacciones cruzadas entre ellos. Por lo tanto, todos deben evitarse en caso de que exista historia de reacción previa asociada a los AINE.

\section{Antibióticos $\beta$-lactámicos y otros}

Los antibióticos son causa frecuente de reacciones adversas durante procedimientos quirúrgicos.

Entre todos los grupos de antibióticos, los $\beta$-lactámicos son los más comúnmente relacionados; las aminopenicilinas y las cefalosporinas son responsables de $70 \%$ de todas las reacciones perioperatorias causadas por los antibióticos. A pesar de esto, la incidencia de las reacciones ha disminuido ostensiblemente en los últimos años.

El grupo de los $\beta$-lactámicos incluye las penicilinas, aminopenicilinas, cefalosporinas, carbapenems y los monobactanes; en la mayoría de los casos, las manifestaciones son mediadas por $\operatorname{IgE}[1-7]$.

Existen reactivos estandarizados para practicar pruebas cutáneas con suficiente valor para predecir futuras reacciones. La dosificación de IgE in vitro también está disponible para las penicilinas y aminopenicilinas. La limitación de este método son los falsos negativos y la inexistencia de reactivos para detectar a presencia de 
IgE específica de los determinantes secundarios de las penicilinas, que son los responsables conocidos de la mayoría de las reacciones anafilácticas.

Las sulfas no se utilizan tan frecuentemente en pacientes quirúrgicos; sin embargo, son medicamentos inmunogénicos que pueden estar relacionados con reacciones graves. La mayoría de las reacciones son desencadenadas por los metabolitos de las sulfas. La realización de pruebas cutáneas no posee validez, como ya fue demostrado en una serie de estudios clínicos, excepto en casos en que exista participación de mecanismos celulares en los cuales la prueba cutánea está indicada.

Otros antibióticos, como las quinolonas y los macrólidos, son poco inmunogénicos. No obstante, se han descrito casos esporádicos de reacciones adversas.

La vancomicina es un antibiótico utilizado con relativa frecuencia en ambientes quirúrgicos. Una reacción adversa común a este medicamento es el llamado síndrome del hombre rojo. Este fenómeno es secundario a la degranulación directa de los mastocitos, sin la presencia de un anticuerpo específico. Generalmente, se relaciona con la velocidad de infusión y en su gran mayoría puede ser prácticamente abolido al infundir el medicamento lentamente. Se pueden usar antihistamínicos y corticosteroides antes de su administración[5-12].

\section{Expansores plasmáticos (coloides)}

Los coloides son causa de $4 \%$ de todas las reacciones anafilácticas intraoperatorias; el $20 \%$ de ellas son graves y ocurren 20 minutos después de iniciar la infusión. Sin embargo, la incidencia puede variar de acuerdo con el agente relacionado: gelatina, almidón, dextrán o albúmina[5-7].

La mayoría de los coloides sintéticos se relacionan con la producción de reacciones anafilácticas, con un mecanismo inmunológico definido.

En casos de reacción a la gelatina, al paciente se le debe orientar para que excluya cualquier sustancia que contenga esta proteína, luego de un adecuado diagnóstico con prueba cutánea, IgE específica o pruebas de activación de basófilos. El paciente también debe recibir orientación especializada en relación con las vacunas que pueden contener gelatina[14].

El dextrán puede causar reacciones mediadas por IgG. La técnica de medición de los anticuerpos IgG y las pruebas cutáneas no están establecidas.

Existen relatos de anafilaxia por albúmina. La alergia al huevo no es contraindicación para la administración de albúmina. 


\section{Anestésicos locales}

Los anestésicos locales son alérgenos incompletos de bajo peso molecular y las reacciones adversas inmediatas que se les imputan son en, su gran mayoría, reacciones vasovagales, tóxicas, psicógenas o efectos colaterales de los vasoconstrictores asociados, como la epinefrina. Eventualmente, las manifestaciones clínicas sugieren una reacción alérgica inmediata. La mayoría de los resultados reportados demuestran que este tipo de efecto es extremamente raro[2, 3, 12].

Existen dos grupos principales de anestésicos locales: los ésteres del ácido benzoico y los del grupo amida.

Con base en estudios con pruebas de contacto, se sabe que los anestésicos del grupo éster reaccionan de forma cruzada entre sí, pero no reaccionan con los anestésicos del grupo amida. Los medicamentos de este último grupo no reaccionan cruzadamente entre sí y son menos sensibilizadores[12].

Muchos pacientes rotulados equivocadamente como alérgicos a los anestésicos locales, evitan procedimientos médicos y odontológicos por desconocimiento o subdiagnóstico, y muchas veces son sometidos a procedimientos sin anestesia. Es posible la realización de pruebas de provocación in vivo, durante las cuales el paciente es expuesto a concentraciones y volúmenes gradualmente mayores del anestésico, con el fin de comprobar la sensibilidad real y poder dar una alternativa adecuada.

\section{Protamina}

La protamina es una proteína de bajo peso molecular utilizada para revertir el efecto anticoagulante de la heparina, principalmente durante cirugías cardíacas.

Algunas reacciones secundarias que se le atribuyen pueden poseer mecanismo inmunológico relacionado o no poseerlo[3].

Los pacientes diabéticos, que utilizan insulina de liberación lenta, ya están sensibilizados y pueden presentar mayor riesgo de reacciones adversas.

El valor diagnóstico positivo de la prueba cutánea no es bueno, pero, existe dosificación de IgE específica.

\section{Ocitocina}

Es una hormona sintética que estimula la contracción uterina, raramente es causa de anafilaxia y el diagnóstico se hace con una prueba cutánea. Es importante recordar que, cuando se administra en dosis altas y por vía intravenosa, puede causar hi- 
potensión, síntoma que se tiene que evaluar para diferenciarlo del choque anafiláctico[3-14].

\section{Látex}

La alergia al látex es la segunda causa de reacción anafiláctica durante los procedimientos quirúrgicos[5, $12,13]$.

Los grupos que presentan mayor riesgo son los niños con espina bífida o anomalías congénitas sometidos a múltiples procedimientos quirúrgicos, y los profesionales de la salud y trabajadores de la industria del caucho. La mayor parte de los insumos de los hospitales, como guantes, catéteres y sondas, contienen látex y la exposición repetitiva por contacto directo o por inhalación, en un evento posterior, podría ser fatal[13].

Actualmente, se realizan intervenciones para la reducción del riesgo de los individuos expuestos, como el cambio de guantes de látex por guantes de polímeros sintéticos, al igual que la práctica de los procedimientos en salas "libres de látex". A pesar de estos esfuerzos y de tener ambientes "libres de látex[7], existen grandes dificultades que conllevan riesgos. En estos casos, todavía se indica la preparación profiláctica con medicaciones previas a la anestesia, sin que con esto se garantice que no ocurran reacciones adversas[14].
Existen extractos para la realización de pruebas cutáneas de lectura inmediata, con buen valor diagnóstico; también, se utiliza la técnica del prick-to-prick, en la cual se realiza la puntura en el propio guante de látex, seguida de la puntura cutánea. Se indica el uso de guantes de, por lo menos, dos lotes diferentes y la dosificación de IgE específica in vitro, que está disponible comercialmente. Cuando la prueba cutánea de lectura inmediata es negativa, está indicado proseguir con la prueba de provocación[13].

\section{Manifestaciones clínicas}

Las manifestaciones clínicas pueden diferir según el grupo o los grupos farmacológicos, su vía de utilización y los factores de riesgo individuales. Las reacciones de hipersensibilidad alérgicas pueden variar en la presentación y en la intensidad, pudiendo manifestarse como cuadros leves, esencialmente con síntomas cutáneos, hasta el choque y la muerte.

La presentación clínica más frecuentemente relatada y de mayor gravedad durante los procedimientos quirúrgicos, es la anafilaxia. No es posible diferenciar clínicamente entre una reacción anafiláctica y la anafilaxia que no es alérgica, pues los síntomas son muy semejantes. La anafilaxia puede ocurrir en cualquier momento del procedimiento quirúrgi- 
co y su progresión puede ser rápida o lenta. Cerca de $90 \%$ de las reacciones se presentan en pocos minutos después de la infusión intravenosa del agente responsable[10].

Las reacciones pueden clasificarse según el tiempo de evolución, como sigue.

Reacciones inmediatas. Se presentan minutos después de la administración del fármaco e incluyen las manifestaciones de anafilaxia. Es la forma de presentación más importante durante los procedimientos quirúrgicos. El $65 \%$ de las muertes por anafilaxia en el intraoperatorio ocurren en los primeros cinco minutos después de la inducción anestésica.

Reacciones aceleradas. Se presentan entre una y tres horas después de la administración del fármaco $\mathrm{y}$, frecuentemente, se manifiestan como urticaria y angiedema. Generalmente, están asociadas al uso de medicamentos en el posoperatorio.

Reacciones tardías. Se presentan después de tres días o más del inicio de la terapia e incluyen eritema cutáneo, fiebre, enfermedad del suero y, menos comúnmente, alteraciones pulmonares, hepáticas, renales o hematológicas. Son reacciones poco asociadas al uso de anestésicos locales o generales.
Los síntomas se pueden clasificar según la gravedad de la anafilaxia, como sigue[6]:

Grado I: síntomas cutáneos, eritema generalizado, urticaria y angiedema

Grado II: síntomas cutáneos, hipotensión, taquicardia y manifestaciones respiratorias, sin riesgo para la vida

Grado III: choque, taquicardia o bradicardia, arritmia, broncoespasmo, con riesgo para la vida

Grado IV: paro cardiorrespiratorio

Grado V: muerte

Generalmente, la anafilaxia grado I no se comprueba durante la cirugía con la utilización de anestésicos generales. La anafilaxia grado II muchas veces se enmascara por el uso de fármacos que mimetizan tales manifestaciones.

\section{Evaluación de la reacción de hipersensibilidad alérgica}

Todo paciente que presente una reacción durante un procedimiento quirúrgico, debe ser investigado posteriormente con el fin de confirmar un posible mecanismo inmunológico relacionado. Se debe identificar el medicamento responsable y detectar posibles reacciones cruzadas, como en 
el caso de los relajantes musculares. Esta investigación debe ser hecha por un alergólogo o inmunólogo clínico. Sin embargo, los datos entregados por el anestesiólogo en la ficha anestésica son imprescindibles para una adecuada evaluación.

La historia clínica es muy importante y se constituye en la principal fuente de información. Todos los medicamentos administrados antes, durante el procedimiento quirúrgico o después de él, la caracterización de las manifestaciones clínicas, el intervalo entre la administración de los medicamentos y el desarrollo de la reacción adversa, deben documentarse, así como la conducta por seguir y la evolución del cuadro clínico.

En todos los casos de reacciones durante procedimientos, la alergia al látex debe ser investigada.

En caso de que exista historia de eccema de contacto, está indicada la realización de la prueba cutánea de contacto (prueba de parche o tardía).

Cuando la historia es sugestiva de anafilaxia clínica, pueden utilizarse métodos de investigación in vitro, como dosificación de triptasa sérica, dosificación de IgE específica o activación de basófilos in vivo, así como la prueba de puntura o intradérmica $\mathrm{y}$, en casos específicos, la provocación. Cada método tiene una indica- ción específica según el caso. Cada uno debe analizarse individualmente.

\section{Triptasa sérica}

La triptasa es un mediador liberado luego de la degranulación de los mastocitos. La elevación de la fracción beta de estos mediadores sugiere la presentación de una reacción anafiláctica. Sin embargo, los niveles normales no la excluyen. El objetivo de la determinación es comprobar un evento anafiláctico, razón por la cual la muestra de sangre debe tomarse hasta dos horas después del inicio de la reacción[3].

\section{Dosificación de IgE específica}

La dosificación de la IgE específica in vitro por radioinmunoensayo, puede ayudar en la identificación del agente responsable. Por ahora, no está disponible para la mayoría de los medicamentos. Aún existe limitación en los métodos de análisis, por el gran número de falsos negativos encontra$\operatorname{dos}[5]$.

\section{Medicamentos disponibles para dosificación de IgE-específica in vitro}

Alérgenos regulares: amoxaciloil, ampiciloil, gelatina, insulina bovina, porcina y humana, peniciloil $\mathrm{G}$, peniciloil $\mathrm{V}$.

Alérgenos especiales: corticotropina (ACTH), cefaclor, quimopapaína, protamina, succinilcolina, toxoide tetánico. 
Prueba de activación de los basófilos (dosificación de CD 63)

Consiste en la verificación de CD 63 por citometría de flujo. Es un método auxiliar para el diagnóstico etiológico. Los estudios hechos con relajantes musculares han demostrado reproducibilidad clínica, con especificidad de $90 \%$ y sensibilidad de $60 \%$ [5].

\section{Pruebas cutáneas de lectura inmediata}

Las pruebas cutáneas deben practicarse, como mínimo, seis semanas después de la reacción adversa, por la mayor posibilidad de falsos negativos[10]. Siempre debe practicarlas un profesional con experiencia.

Únicamente la penicilina y el látex tienen reactivos para pruebas cutáneas disponibles comercialmente. Otros medicamentos deben diluirse conforme a protocolos ya establecidos hasta concentraciones no irritantes, antes de utilizarse. Las pruebas cutáneas intradérmicas son un método auxiliar que evalúa las reacciones inmediatas y tardías para determinados medicamentos [5].

Los resultados negativos no descartan la posibilidad de una futura reacción por el medicamento probado.

\section{Pruebas de provocación}

Las pruebas de provocación consisten en la administración controlada del fármaco, con el objetivo de diagnosticar una reacción de hipersensibilidad a la sustancia probada. La prueba debe ser siempre indicada por un especialista en ambiente hospitalario, con equipo disponible para reanimación y equipo médico entrenado, dado que son pruebas potencialmente peligrosas[7-15] que pueden poner en riesgo la vida del paciente. Unas contraindicaciones relativas son el embarazo y enfermedades concomitantes, como infecciones agudas, asma descompensada, cardiopatía, nefropatía o hepatopatía. Están contraindicadas en casos de anafilaxia grave; sin embargo, el riesgo beneficio debe evaluarse individualmente, principalmente cuando la prueba cutánea o las pruebas in vitro no aclaren el diagnósti$\operatorname{co}[15]$.

A pesar de que la prueba cutánea es la de referencia para el diagnóstico de las reacciones adversas a fármacos, su interpretación es difícil, principalmente cuando existen quejas subjetivas, lo que posibilita resultados falsos. El mecanismo fisiopatológico y las reacciones pueden no ser completamente patognomónicos, pues pueden no existir factores concomitantes para que se presente la reacción adversa.

\section{Estrategia de investigación preoperatoria}

A pesar del valor pronóstico, para la mayoría de las pruebas diagnósticas 
utilizadas en la investigación de reacciones secundarias a medicamentos, no existen pruebas actuales que validen la realización de una tamización previa a la cirugía en la población general. Tampoco existe la indicación de practicar estas pruebas en individuos atópicos o sensibilizados a otras sustancias que no vayan a utilizarse durante el acto quirúrgico. Se debe hacer investigación en situaciones específicas, como las siguientes[2, 4, 6, 13-17]:

- pacientes sensibilizados a agentes anestésicos o al látex,

- pacientes con historia previa de reacciones durante procedimientos quirúrgicos o después de ellos, con anestesia general (broncospasmo, hipotensión o angiedema),

- reacciones posteriores al uso de anestésicos locales y

- pacientes pertenecientes a los grupos de riesgo para sensibilización al látex.

\section{Conclusión}

Las reacciones de hipersensibilidad a los anestésicos locales y generales, y a otros medicamentos utilizados durante los procedimientos quirúrgicos, aún representan grandes desafíos en la práctica clínica. Continúan siendo motivo de estudio y profundización para los profesionales de la salud, quienes encaminan sus esfuerzos a hacer diagnósticos pertinentes que proporcionen óptimos resultados con mínimos riesgos para la vida de los pacientes.

Nuestro objetivo es continuar avanzando en el conocimiento científico, minimizar costos y evitar que tales reacciones adversas ocurran o se repitan.

Ejemplos de agentes causantes de anafilaxia intraoperatoria y procedimientos médicos

Relajantes musculares

Succunilcolina (suxametonio), atracurio, vecuronio, pancuronio Agentes inductores Barbitúricos, etomidato, propofol Narcóticos

Fentanilo, meperidina, morfina Coloides Gelatina, dextrán, albúmina Antibióticos $\beta$-lactámicos, vancomicina, quinolonas Antiinflamatorios no esteroideos Medios de contraste Derivados de la sangre Látex Guantes, catéteres, sondas, etc. Otros Protamina, manitol

Ejemplos de agentes causantes de anafilaxia intraoperatoria y procedimientos médicos 
Relajantes musculares

Succunilcolina (suxametonio), atracurio, vecuronio, pancuronio

Agentes inductores

Barbitúricos, etomidato, propofol

Narcóticos

Fentanilo, meperidina, morfina

\section{Coloides}

Gelatina, dextrán, albúmina

Antibióticos

$\beta$-lactámicos, vancomicina, quinolonas

Antiinflamatorios no esteroideos

Medios de contraste

Derivados de la sangre

Látex

Guantes, catéteres, sondas, etc.

Otros

Protamina, manitol

\section{Bibliografía}

1. Lieberman P. Anaphylactic reactions during surgical and medical procedures. JACI. 2002;110(Suppl.2):S64-9.

2. Johansson SGO, Bieber T, Dahl R, Friedeman P, Lanier BQ, Lockey RF. Revised nomenclature for allergy for global use: Report of the nomenclature review committee of the World Allergy Organization, October 2003. JACI. 2003;13:832-6.

3. Ebo DG, Fisher MM, Hagendorens MM, Bridts CH, Stevens WJ. Anaphylaxis during anesthesia: diagnostic approach. Allergy. 2007;62:471-87.

4. Laxenaire MC, Mertes PM. Anaphylaxis during anesthesia. Results of two- year survey in France. Br J Anesth. 2001;87:549-58.

5. Fisher MM. The preoperative detection of risk of anaphylaxis during anesthesia. Anaesth Intensive Care. 2007; 35:899-902.

6. Mertes PM, Laxenaire MC. Allergic reactions occurring during anesthesia. Eur J Anaesthesiol. 2002;19:240-62.

7. Mertes PM, Laxenaire MC, Lienhart A, Aberer W, Ring J, Pichler WJ. Reducing the risk of anaphylaxis during anesthesia: Guidelines for a clinical practice. $J$ Invest Allergol Clin Immunol. 2005;15:91-101.

8. Lieberman P. Epidemiology of anaphylaxis. Curr Opin Allergy Clin Immunol. 2008;8:316-20.

9. Demoly P, Viola M, Rebelo Gomes E, Romano A. Epidemiology and causes of drug hypersensitivity. In: Pichler WJ, editor. Drug hypersensitivity. Basel: Karger. 2007;2-17.

10. Mertes PM, Laxenaire MC. Allergy and anaphylaxis in anesthesia. Minerva Anaesth. 2004;70:285-91.

11. Licardi G, Lobefalo G, DiFlorio E, Di Iorio C, Occhiochiuso L, Romano L. Strategies for the prevention of asthmatic, anaphylactic and anaphylactoid reactions during the administration of anesthetics and/or contrast media. $J$ Invest Allergol Clin Immunol. 2008; 18:1-11.

12. Thong BYH, Chan Y. Anaphylaxis during surgical and interventional procedure. Ann Allergy Clin Immunol. 2004;92:619-28.

13. Bernard YHT. Anaphylaxis during surgical and interventional procedures. Ann Allergy Asthma Immunol. 2004; 92:619-28. 
14. Fisher MM, Doig GS. Prevention of anaphylactic reactions to anesthetic drugs. Drug Saf. 2004;27:393-410.

15. Aberer W, Bircher A, Romano A, Blanca M, Campi P, Fernandez J. Drug provocation testing in diagnosis of drug hypersensitivity reactions: General considerations. Allergy. 2003;58:854-63.
16. Elliot BA. Latex allergy: The perspective from the surgical suite. JACI. 2002;110:117-20.

17. Gruchalla R. Understanding drug allergies. J Allergy Clin Immunol. 2000; 105(Suppl.):S637-44. 